Discussion Paper No. 927

\title{
NOTIONS OF ANONYMITY \\ FOR OBJECT ASSIGNMENT: \\ IMPOSSIBILITY THEOREMS
}

\author{
Hikaru Kondo \\ Shigehiro Serizawa
}

March 2015

The Institute of Social and Economic Research

Osaka University

6-1 Mihogaoka, Ibaraki, Osaka 567-0047, Japan 


\title{
Notions of anonymity for object assignment: impossibility theorems*
}

\author{
Hikaru Kondo \\ Graduate School of Economics, Osaka University \\ 1-7, Machikane-yama, Toyonaka, Osaka, 560-0043, Japan \\ E-mail: nge009kh@student.econ.osaka-u.ac.jp \\ and \\ Shigehiro Serizawa \\ Institute of Social and Economic Research, Osaka University \\ 6-1, Mihogaoka, Ibaraki, 567-0047, Japan \\ E-mail: serizawa@iser.osaka-u.ac.jp
}

February 27, 2015

\begin{abstract}
We search for impartiality in the allocation of objects when monetary transfers are not possible. Our main focus is anonymity. The standard definition requires that if agents' names are permuted, their assignments should be permuted in the same way. Since no rule satisfies this definition in this model, we introduce weaker variants, "anonymity on distinct preferences," "pairwise-anonymity on distinct preferences," "pairwise-anonymity on distinct profiles," and "independence of others' permutations." We show that for more than two agents and two objects, no rule is pairwise-anonymous on distinct preferences and Pareto-efficient (Theorem 1), no rule is pairwise-anonymous on distinct preferences and independent of others' permutations (Theorem 2), and no rule is pairwise-anonymous on distinct profiles and strategy-proof (Theorem 3). These results suggest that introducing randomization to object allocation problems is almost inevitable for achieving impartiality.
\end{abstract}

Keywords: impartiality, anonymity, indivisible goods

JEL Classification Numbers: C78, D71, D78

*First, we thank William Thomson for his careful reading and detailed and useful comments. We also thank Masaki Aoyagi, Agustin Bonifacio, Patrick Harless, Kazuhiko Hashimoto, Morimitsu Kurino, Noriaki Matsushima, Anup Pramanik, James Schummer, Tayfun Sonmez, Wataru Tamura, Masashi Tanaka, Utku Unver, Jun Wako, and participants at Frontiers of Market Design, Osaka University Applied Microeconomic Theory Workshop, 11th Meeting of Society for Social Choice and Welfare and Osaka-Rochester Theory Workshop for their helpful comments. We are especially grateful to Shuhei Morimoto and Koji Takamiya for detailed comments and helpful suggestions. This work is supported by Grant-in-Aid for JSPS Fellows. All remaining errors are our own. 


\section{Introduction}

We consider the problem of assigning a number of indivisible goods, called "objects," to an equal number of agents when monetary transfers are not possible. ${ }^{1}$ Examples include a manager assigning tasks to workers, or a government assigning housing units to low-income applicants. Each agent has a strict preference ${ }^{2}$ over objects and receives exactly one object. A rule is a function from a class of preference profiles to the set of feasible allocations that assigns objects to agents.

In the context of such problems, people are often sensitive to impartiality of rules. Thus, it is important to achieve impartiality. One way to achieve impartiality is using randomization, such as with the "random priority" (RP) rule. ${ }^{3}$ However, the following question arises: "Is randomization necessary for achieving impartiality?" In other words, "Are there ways to achieve impartiality other than randomization?" Motivated by this question, we explore the possibilities of achieving impartiality in a deterministic environment.

"Anonymity" is a requirement commonly used to embody impartiality. Anonymity states that allocations are independent of agents' names. Its standard mathematical formulation is as follows: if agents' names are permuted, their assignments should be permuted in the same way. We refer to this definition as "standard anonymity." Here, standard anonymity implies that when several agents have the same attribute (preference), they are all assigned the same object, which is impossible in our model. However, the following example suggests that impartiality may be achieved by weakening the standard anonymity requirement.

ExAmple 1: There are two objects, $a$ and $b$, and two agents, 1 and 2 . Consider the following rule. If the two agents prefer different objects, each receives the one he prefers. If both prefer $a$ to $b$, agents 1 and 2 receive $a$ and $b$, respectively; if both prefer $b$ to $a$, then agents 1 and 2 receive $a$ and $b$, respectively.

This rule does not satisfy standard anonymity, because the allocations depend on the agents' names when both agents have the same preferences. However, it still appears to be impartial. At the two profiles in which both agents have distinct preferences, both obtain their favorite objects. At the other two profiles, one of the agents must be chosen as the recipient of his favorite object. In one such profile, agent 1 is chosen, and in the other, agent 2 is chosen. Therefore, each agent receives his favorite in an equal number of preference profiles. Although the rule is not impartial at each profile, it seems impartial if we look at the collection of profiles. Thus, it may be called "impartial across profiles." 4

Based on this observation, we propose several notions of anonymity. "Anonymity on distinct preferences" imposes the same requirement as standard anonymity, except that we do not permute agents who have the same preferences. "Pairwise-anonymity on distinct preferences" requires that when two agents have distinct preferences and their preferences are transposed, their assignments should be transposed and nothing is required of the other agents' assignments.

\footnotetext{
${ }^{1}$ The formal study of object allocation problems originates in Shapley and Scarf (1974).

${ }^{2}$ In practice, people may be indifferent among several objects. In this model, however, allowing indifference makes it difficult to construct desirable rules (Ehlers (2002)).

${ }^{3}$ The RP rule works as follows: For each agent priority, each agent receives his favorite object among the remaining objects. If there are $n$ agents, there are $n$ ! possible agent priorities. Then, compute the average probability of all allocations determined by each priority.

${ }^{4}$ Thomson (2012) makes the same point.
} 
"Pairwise-anonymity on distinct profiles" imposes the same requirement as pairwise-anonymity on distinct preferences, except that we impose it only on profiles in which all agents have distinct preferences. Again, nothing is required of other agents' assignments. "Independence of others' permutations" requires that if, for example, the preferences of agents other than agent $i$ are permuted but agent $i$ 's preference remains constant, agent $i$ 's assignment should not change. Anonymity on distinct preferences is stronger than both pairwise-anonymity on distinct preferences and independence of others' permutations, and pairwise-anonymity on distinct preferences is stronger than pairwise-anonymity on distinct profiles.

The rule in Example 1 has other interesting properties. First, it is Pareto-efficient: for each preference profile, it chooses an allocation such that there is no other allocation at which each agent is at least as well off and at least one agent is better off. Second, it is strategy-proof: for each preference profile, truth-telling is a weakly dominant strategy in the direct revelation game associated with the rule.

This rule shows the compatibility of anonymity on distinct preferences, Pareto-efficiency, and strategy-proofness for two agents and two objects. Naturally, this generates questions about the general case, which is our focus. Unfortunately, our answers are negative. We show that for more than two agents and two objects, no rule is pairwise-anonymous on distinct preferences and Pareto-efficient (Theorem 1). Furthermore, no rule is pairwise-anonymous on distinct preferences and independent of others' permutations (Theorem 2). Finally, no rule is pairwise-anonymous on distinct profiles and strategy-proof (Theorem 3). These results suggest that to achieve impartiality, introducing randomization into object allocation problems is almost inevitable. In this sense, these results motivate the research on probabilistic assignment problems.

Let us discuss the related literature concerning anonymity for discrete resource allocation problems. Consider the case in which not receiving an object is an option, termed "null object." In such a case, we can construct standard anonymous rules by sacrificing Pareto-efficiency (Kesten and Yazici (2012)). ${ }^{5}$ In their study, the null object is not necessarily ranked worst. ${ }^{6}$ Moreover, rules satisfying standard anonymity can exist in the model without the null object. If we assume that agents initially own the objects, there are standard anonymous rules (Miyagawa (2002)). Miyagawa shows that the core and the no-trade rules are characterized by standard anonymity and some other properties. ${ }^{7}$ Then, anonymity in these models is not as demanding as that in our model. In the model of Kesten and Yazici (2012), the rule that assigns the null object to all agents at all profiles is obviously standard anonymous. In the standard object exchange model, standard anonymous rules can also be defined.

No-envy and equal treatment of equals are also well-known fairness requirements. No-envy says that no agent should prefer someone else's assignment to his own, and equal treatment of equals says that agents with the same preferences should be assigned objects between which they are indifferent. No rule satisfies standard definitions of these axioms in our model. Then, in the hope of achieving fairness, probabilistic rules are considered. However, impossibility results for the probabilistic assignment concerning fairness may still exist. If agents have a von Neumann-Morgenstern (vNM) utility function, no rule satisfies equal treatment of equals

\footnotetext{
${ }^{5}$ Although their main fairness requirement is no-envy, some of their results also hold if this requirement is replaced by anonymity.

${ }^{6}$ The null object may play an important role as an option. See Kesten and Kurino (2013).

${ }^{7}$ The core can be characterized even without anonymity. See, for example, Ma (1994) or Takamiya (2001).
} 
together with (ex-ante) Pareto-efficiency and strategy-proofness (Zhou (1990)). Instead of the vNM utility function, we can introduce the notion of stochastic dominance (sd) into probabilistic assignment to compare lotteries. Even in this setting, no rule satisfies equal treatment of equals, (sd) Pareto-efficiency and (sd) strategy-proofness (Bogomolnaia and Moulin (2001)). Because (sd) no-envy is stronger than equal treatment of equals, (sd) no-envy is also incompatible with the other two requirements. Although we find such negative results, we may find better results if we weaken some properties. For example, the RP rule is anonymous together with (sd) strategy-proof and (ex-post) Pareto-efficient.

When fairness requirements are dropped, rules satisfying other desirable properties are identified. They use hierarchies of agents. See, for example, Svensson (1999) and Papai (2000a) for the single-unit demand case; Papai (2000b) and Hatfield (2009) for the multi-unit demand case; and Abdulkadiroglu and Sonmez (1999) for the case in which some agents initially own objects.

The remainder of this paper is organized as follows: In Section 2, we provide formal definitions of the model and basic requirements. In Section 3, we define three of our new notions and show some results concerning them. In Section 4, we define "pairwise-anonymity on distinct profiles" and establish possibility and impossibility results. In Section 5, we show the independence of the axioms of the theorems. In Section 6, we provide some concluding remarks.

\section{Model and definitions}

We denote the set of agents by $N=\{1,2, \cdots, n\}(n \geq 2)$ and the set of objects by $K$, where $|K|=n .{ }^{8}$ Each agent $i \in N$ has a complete, transitive, and antisymmetric preference $R_{i}$ over $K{ }^{9}$ We denote the set of all preferences by $\mathcal{R}$. We denote the strict preference associated with $R_{i}$ by $P_{i}$, that is, $a P_{i} b$ if and only if $a R_{i} b$ and $a \neq b$. We denote the top-ranked object for $R_{i}$ by $t\left(R_{i}\right)$ and the second-ranked object by $s t\left(R_{i}\right)$. We represent a preference $R_{i}$ as follows:

$$
R_{i}: a b c d \cdots
$$

where $a, b, c, d, \cdots \in K$. This means that $a=t\left(R_{i}\right), b=s t\left(R_{i}\right)$, and so on. Given $R_{i} \in \mathcal{R}$ and $a \in K$, we denote the lower contour set of $R_{i}$ at $a$ as $L\left(R_{i}, a\right)=\left\{k \in K \mid a R_{i} k\right\}$.

For each $i \in N, x_{i}$ is the object assigned to agent $i$. A feasible allocation is a list of objects $x=\left(x_{i}\right)_{i \in N}$ such that for each pair $\{i, j\} \subset N, x_{i} \neq x_{j}$. Let $X$ be the set of all feasible allocations. Let $R=\left(R_{i}\right)_{i \in N}$. For each pair $\{i, j\} \subset N, R_{-i}$ denotes a preference profile for $N \backslash\{i\}$, and $R_{-i j}$ denotes a preference profile for $N \backslash\{i, j\}$. We denote the set of all preference profiles for $N$ by $\mathcal{R}^{N}$.

An (assignment) rule $f$ is a function from $\mathcal{R}^{N}$ to $X$. For each pair $\{i, j\} \subset N$ and each $R \in \mathcal{R}^{N}$, we denote the object assigned to $i$ at $f(R)$ as $f_{i}(R)$, and $\left(f_{i}(R), f_{j}(R)\right)$ as $f_{i, j}(R)$.

Next, we introduce two properties of rules. Let $f$ be a rule and $R \in \mathcal{R}^{N}$. First, for each preference profile, a rule should choose an allocation such that there is no allocation at which each agent is as well off and at least one agent is better off. An allocation $\hat{x} \in X$ Paretodominates $x$ at $R$ if for each $i \in N, \hat{x}_{i} R_{i} x_{i}$ and for some $j \in N, \hat{x}_{j} P_{j} x_{j}$.

\footnotetext{
${ }^{8}$ Most of our results hold even if $|N|<|K|$.

${ }^{9} \mathrm{~A}$ preference $R_{i}$ is complete if for each $a, b \in K, a R_{i} b$ or $b R_{i} a . R_{i}$ is transitive if for each $a, b, c \in K, a R_{i} b$ and $b R_{i} c$ imply $a R_{i} c$. $R_{i}$ is antisymmetric if for each $a, b \in K, a R_{i} b$ and $b R_{i} a$ imply $a=b$.
} 
Pareto-efficiency: For each $R \in \mathcal{R}^{N}$, no allocation Pareto-dominates $f(R)$ at $R$.

Next, for each preference profile, truth-telling should be a weakly dominant strategy for each agent in the direct revelation game associated with the rule.

Strategy-proofness: For each $R \in \mathcal{R}^{N}$, each $i \in N$ and each $\hat{R}_{i} \in \mathcal{R}, f_{i}(R) R_{i} f_{i}\left(\hat{R}_{i}, R_{-i}\right)$.

\section{Several notions of anonymity}

The principle of anonymity is that allocations should be determined independently of the agents' names. In this section, we introduce several variants. Given a profile $R$ and a permutation $\pi: N \rightarrow N$, let $R^{\pi} \equiv\left(R_{\pi(i)}\right)_{i \in N}$ be the profile obtained by permuting the agents' names according to $\pi$. Let $\Pi_{\text {dist }}(R) \equiv\left\{\pi: \forall i \in N, \pi(i)=j \neq i \Rightarrow R_{i} \neq R_{j}\right\}$ denote the permutations such that only agents with distinct preferences are permuted. The next requirement is that if agents are permuted according to $\pi \in \Pi_{\text {dist }}(R)$, the assignments should be permuted in the same way.

Anonymity on distinct preferences: For each $R \in \mathcal{R}^{N}$, each $\pi \in \Pi_{\text {dist }}(R)$, and each $i \in N$, $f_{i}\left(R^{\pi}\right)=f_{\pi(i)}(R)$.

For most models, anonymity implies the axiom called equal treatment of equals. ${ }^{10}$ Here, however, anonymity on distinct preferences is logically independent of equal treatment of equals. Anonymity on distinct preferences also requires that if a permutation does not affect some agents, their assignments should not change. Example 1 in Section 1 satisfies anonymity on distinct preferences in addition to Pareto-efficiency and strategy-proofness. That example shows that our impossibility results are not trivial.

We define two other anonymity axioms. If two agents have distinct preferences and their preferences are transposed, their assignments should also be transposed.

Pairwise-anonymity on distinct preferences: For each $R \in \mathcal{R}^{N}$ and each pair $\{i, j\} \subset N$, $R_{i} \neq R_{j}$ implies $f_{i}\left(R_{i}, R_{j}, R_{-i j}\right)=f_{j}\left(\hat{R}_{i}, \hat{R}_{j}, R_{-i j}\right)$ and $f_{j}\left(R_{i}, R_{j}, R_{-i j}\right)=f_{i}\left(\hat{R}_{i}, \hat{R}_{j}, R_{-i j}\right)$, where $\hat{R}_{i}=R_{j}$ and $\hat{R}_{j}=R_{i}$.

Anonymity on distinct preferences implies pairwise-anonymity on distinct preferences. Pairwiseanonymity on distinct preferences allows the existence of agents whose preferences are not permuted but whose assignments change.

Given $i \in N$, let $\Pi_{i}$ denote the set of permutations such that only agents other than $i$ are permuted. That is, $\pi \in \Pi_{i}$ implies $\pi(i)=i$. The following condition states that agent $i$ 's assignment should not change even if others' names are permuted.

Independence of others' permutations: For each $R \in \mathcal{R}^{N}$, each $i \in N$, and each $\pi \in \Pi_{i}$, $f_{i}(R)=f_{i}\left(R^{\pi}\right)$.

Anonymity on distinct preferences also implies independence of others' permutations. When $n \leq 3$, any rule is independent of others' permutations. We turn to our impossibility results.

Theorem 1: Let $n \geq 3$. Then, no rule is pairwise-anonymous on distinct preferences and Pareto-efficient.

Proof: Let $f$ be a rule satisfying the axioms of the theorem. Let $R \in \mathcal{R}^{N}$ be such that

\footnotetext{
${ }^{10}$ Equal treatment of equals: For each $R \in \mathcal{R}^{N}$ and each pair $\{i, j\} \subset N$ such that $R_{i}=R_{j}, f_{i}(R)=f_{j}(R)$.
} 
(a) $R_{1}=R_{2}$

(b) for each $i \in N \backslash\{1,2\}, t\left(R_{i}\right) \neq t\left(R_{1}\right)$ and $t\left(R_{i}\right) \neq s t\left(R_{1}\right)$, and

(c) for each $i, j \in N \backslash\{1,2\}, t\left(R_{i}\right) \neq t\left(R_{j}\right)$.

Step 1:

$$
\begin{aligned}
& f(R)=\left(t\left(R_{1}\right), \operatorname{st}\left(R_{2}\right), t\left(R_{3}\right), t\left(R_{4}\right), \cdots, t\left(R_{n}\right)\right) \equiv x^{1} \text { or } \\
& f(R)=\left(\operatorname{st}\left(R_{1}\right), t\left(R_{2}\right), t\left(R_{3}\right), t\left(R_{4}\right), \cdots, t\left(R_{n}\right)\right) \equiv x^{2}
\end{aligned}
$$

By conditions (a), (b), and (c), $x^{1}$ and $x^{2}$ are both feasible. Let $x \in X \backslash\left\{x^{1}, x^{2}\right\}$. We show that $x$ is Pareto-dominated by $x^{1}$ or $x^{2}$. Because $x \neq x^{1}$ and $x \neq x^{2}$, there are two cases:

Case 1: There is $i \in\{1,2\}$ such that $\operatorname{st}\left(R_{i}\right) P_{i} x_{i}$

If $i=1, x_{1}^{2}=\operatorname{st}\left(R_{1}\right) P_{1} x_{1}$, and for each $j \in N \backslash\{1\}, x_{j}^{2}=t\left(R_{j}\right) R_{j} x_{j}$. This means that $x^{2}$ Pareto-dominates $x$. If $i=2, x^{1}$ Pareto-dominates $x$.

Case 2: There is $j \in N \backslash\{1,2\}$ such that $x_{j} \neq t\left(R_{j}\right)$.

By condition (a), $x_{1} \neq t\left(R_{1}\right)$ or $x_{2} \neq t\left(R_{2}\right)$. If $x_{1} \neq t\left(R_{1}\right), x^{2}$ Pareto-dominates $x$. If $x_{2} \neq t\left(R_{2}\right), x^{1}$ Pareto-dominates $x$.

Step 2: We derive a contradiction. By Step 1, we may assume that $f(R)=x^{1}$, because the case $f(R)=x^{2}$ can be treated symmetrically. Let $f_{1}(R)=t\left(R_{1}\right) \equiv a, f_{2}(R)=s t\left(R_{2}\right) \equiv b$, and $f_{3}(R)=t\left(R_{3}\right) \equiv c$. Let $R^{2} \equiv\left(R_{1}, R_{3}, R_{2}, R_{4}, \cdots, R_{n}\right)$ be a profile obtained from $R$ by transposing the preferences of agents 2 and 3. By pairwise-anonymity on distinct preferences and (b), $f_{2}\left(R^{2}\right)=f_{3}(R)=c$ and $f_{3}\left(R^{2}\right)=f_{2}(R)=b$. Moreover, by Pareto-efficiency, for each $i \in N \backslash\{2,3\}, f_{i}\left(R^{2}\right)=t\left(R_{i}\right)$. Thus, $f\left(R^{2}\right)=\left(a, c, b, t\left(R_{4}^{2}\right), \cdots, t\left(R_{n}^{2}\right)\right)$. Similarly, we define $R^{3} \equiv\left(R_{2}^{2}, R_{1}^{2}, R_{3}^{2}, R_{4}^{2}, \cdots, R_{n}^{2}\right)$ from $R^{2}$ by transposing the preferences of agents 1 and 2 , and we find that $f\left(R^{3}\right)=\left(c, a, b, t\left(R_{4}^{3}\right), \cdots, t\left(R_{n}^{3}\right)\right)$. Now, let $R^{4} \equiv\left(R_{3}^{3}, R_{2}^{3}, R_{1}^{3}, R_{4}^{3}, \cdots, R_{n}^{3}\right)$. By pairwise-anonymity on distinct preferences, $f_{1}\left(R^{4}\right)=f_{3}\left(R^{3}\right)=b$. However, $R_{1}=R_{2}$ implies $R^{4}=R$, and thus, $f_{1}\left(R^{4}\right)=f_{1}(R)=a \neq b$. This is a contradiction.

Q.E.D.

Theorem 2: Let $n \geq 3$. Then, no rule is pairwise-anonymous on distinct preferences and independent of others' permutations. ${ }^{11}$

Proof: Let $f$ be a rule satisfying the axioms of the theorem. We derive a contradiction. Let $R \in \mathcal{R}^{N}$ be such that $R_{1}=R_{2} \neq R_{3}, R^{2} \equiv\left(R_{1}, R_{3}, R_{2}, R_{4}, \cdots, R_{n}\right), R^{3} \equiv\left(R_{3}, R_{1}, R_{2}, R_{4}, \cdots\right.$ $\left.\cdot, R_{n}\right)$, and $R^{4} \equiv\left(R_{2}, R_{1}, R_{3}, R_{4}, \cdots, R_{n}\right)$. Then, by pairwise-anonymity on distinct preferences and independence of others' permutations,

$$
\begin{aligned}
f\left(R^{2}\right) & =\left(f_{1}(R), f_{3}(R), f_{2}(R), f_{4}(R), \cdots, f_{n}(R)\right), \\
f\left(R^{3}\right) & =\left(f_{3}(R), f_{1}(R), f_{2}(R), f_{4}(R), \cdots, f_{n}(R)\right), \text { and } \\
f\left(R^{4}\right) & =\left(f_{2}(R), f_{1}(R), f_{3}(R), f_{4}(R), \cdots, f_{n}(R)\right) .
\end{aligned}
$$

However, by $R_{1}=R_{2}$,

\footnotetext{
${ }^{11}$ To be exact, if $n=3$, any rule satisfies independence of others' permutations so that no rule is pairwiseanonymous on distinct preferences.
} 
$f\left(R^{4}\right)=f(R)=\left(f_{1}(R), f_{2}(R), f_{3}(R), f_{4}(R), \cdots, f_{n}(R)\right)$. This is a contradiction. $\quad$ Q.E.D.

Because anonymity on distinct preferences implies pairwise-anonymity on distinct preferences and independence of others' permutations, Corollary 1 follows.

Corollary 1: Let $n \geq 3$. Then, no rule is anonymous on distinct preferences.

\section{Pairwise-anonymity on distinct profiles}

As shown, pairwise-anonymity on distinct preferences is too strong in the model. Therefore, in this section we introduce a weaker condition. Let $\mathcal{R}^{N *} \equiv\left\{R^{\prime} \in \mathcal{R}^{N} \mid \forall i, j \in N, i \neq j \Rightarrow R_{i}^{\prime} \neq\right.$ $R_{j}^{\prime}$ \} denote the set of preference profiles in which no two agents have the same preferences. We call such profiles "distinct profiles." The next requirement is weaker than pairwise-anonymity on distinct preferences: we impose the same condition only on distinct profiles.

Pairwise-anonymity on distinct profiles: For each $R \in \mathcal{R}^{N *}$ and each pair $\{i, j\} \subset N$, $f_{i}\left(R_{i}, R_{j}, R_{-i j}\right)=f_{j}\left(\hat{R}_{i}, \hat{R}_{j}, R_{-i j}\right)$ and $f_{j}\left(R_{i}, R_{j}, R_{-i j}\right)=f_{i}\left(\hat{R}_{i}, \hat{R}_{j}, R_{-i j}\right)$, where $\hat{R}_{i}=R_{j}$ and $\hat{R}_{j}=R_{i}$.

Pairwise-anonymity on distinct preferences implies pairwise-anonymity on distinct profiles. Here, we can also consider the weaker notion of no-envy by imposing the requirement only on $\mathcal{R}^{N *}$. ${ }^{12}$ However, we do not introduce such a condition, because no rule satisfies it in our model.

Pairwise-anonymity on distinct profiles is logically related to other known conditions. The first condition states that there should not be an agent who is always assigned his most preferred object.

Non-dictatorship: There is no agent $i \in N$ such that for each $R \in \mathcal{R}^{N}, f_{i}(R)=t\left(R_{i}\right)$.

The next condition states that each allocation should be chosen for some preference profile. Respect of consumer sovereignty: For each $x \in X$, there exists $R \in \mathcal{R}^{N}$ such that $f(R)=$ $x$.

There are rules that are non-dictatorial, Pareto-efficient, and strategy-proof (see, e.g., Svensson (1999) or Papai (2000)). We establish two facts.

Fact 1: Let $n \geq 3$. If a rule $f$ is pairwise-anonymous on distinct profiles, it is non-dictatorial. Proof: Suppose not. Then, there is $i \in N$ such that for each $R \in \mathcal{R}^{N}, f_{i}(R)=t\left(R_{i}\right)$. Let $R \in \mathcal{R}^{N *}$ be such that there is $j \in N$ such that $j \neq i$ and $t\left(R_{j}\right)=t\left(R_{i}\right)$. Here, $t\left(R_{j}\right) \neq f_{j}(R)$. Then, by pairwise-anonymity on distinct profiles, $f_{i}\left(\hat{R}_{i}, \hat{R}_{j}, R_{-i j}\right)=f_{j}(R) \neq t\left(\hat{R}_{i}\right)$, where $\hat{R}_{i}=R_{j}$ and $\hat{R}_{j}=R_{i}$. However, $f_{i}\left(\hat{R}_{i}, \hat{R}_{j}\right.$, and $\left.R_{-i j}\right)=t\left(\hat{R}_{i}\right)$, because $i$ is the dictator. This is a contradiction.

Q.E.D.

Fact 2: If a rule $f$ is pairwise-anonymous on distinct profiles and independent of others' permutations, it respects consumer sovereignty.

Proof: Let $x \in X$. We show that there is $R \in \mathcal{R}^{N}$ such that $f(R)=x$. Let $R^{0} \in \mathcal{R}^{N *}$. Using pairwise-anonymity on distinct profiles and independence of others' permutations, we find $R$ as follows:

\footnotetext{
${ }^{12}$ No-envy: For each pair $\{i, j\} \subset N$ and each $R \in \mathcal{R}^{N}, f_{i}(R) R_{i} f_{j}(R)$.
} 
Step 1: Constructing $R^{1} \in \mathcal{R}^{N *}$ such that $f_{1}\left(R^{1}\right)=x_{1}$. Let $j \in N$ be such that $f_{j}\left(R^{0}\right)=$ $x_{1}$. If $j=1$, let $R^{1} \equiv R^{0}$ and proceed to the next step. Let $j \neq 1$ and let $R^{1} \equiv\left(R_{j}, R_{2}, \cdots\right.$ $\left.\cdot, R_{j-1}, R_{1}, R_{j+1}, \cdots, R_{n}\right)$ be obtained from $R^{0}$ by transposing the preferences of agents 1 and $j$. Then, by pairwise-anonymity on distinct profiles and independence of others' permutations,

$$
f\left(R^{1}\right)=\left(x_{1}, f_{2}\left(R^{1}\right), \cdots, f_{j-1}\left(R^{1}\right), f_{1}\left(R^{1}\right), f_{j+1}\left(R^{1}\right), \cdots, f_{n}\left(R^{1}\right)\right) .
$$

Step 2: Constructing $R^{2} \in \mathcal{R}^{N *}$ such that $f_{1,2}\left(R^{2}\right)=\left(x_{1}, x_{2}\right)$. Let $j^{\prime} \in N$ be such that $f_{j^{\prime}}\left(R^{1}\right)=x_{2}$. If $j^{\prime}=2$, let $R^{2} \equiv R^{1}$ and proceed to the next step. Let $j^{\prime} \neq 2$ and let $R^{2} \equiv\left(R_{1}^{1}, R_{j^{\prime}}^{1}, \cdots, R_{j^{\prime}-1}^{1}, R_{2}^{1}, R_{j^{\prime}+1}^{1}, \cdots, R_{n}^{1}\right)$ be obtained by transposing $R_{j^{\prime}}^{1}$ and $R_{2}^{1}$. Then, by pairwise-anonymity on distinct profiles and independence of others' permutations,

$$
f\left(R^{2}\right)=\left(x_{1}, x_{2}, f_{3}\left(R^{2}\right), \cdots, f_{j^{\prime}-1}\left(R^{2}\right), f_{2}\left(R^{2}\right), f_{j^{\prime}+1}\left(R^{2}\right), \cdots, f_{n}\left(R^{2}\right)\right) .
$$

Iterating, we obtain $R^{n} \in \mathcal{R}$ such that $f\left(R^{n}\right)=\left(x_{1}, x_{2}, \cdots, x_{n}\right)=x$.

Q.E.D.

We have already seen that no rule is both pairwise-anonymous on distinct preferences and Pareto-efficient. However, there are rules that are pairwise-anonymous on distinct profiles and Pareto-efficient. We define a family of such rules next. Preference priorities and agent priorities are used in the definition. The agent whose preference has the highest priority obtains his mostpreferred object. Then, the agent whose preference has the second-highest priority obtains his most-preferred object among the remaining objects, and so on. If, at some step, several agents have the same preferences, we use agent priorities to break ties. The agent with the highest agent priority among them obtains his most-preferred object among the remaining objects, the agent with the second-highest agent priority obtains his most-preferred object among the remaining objects, and so on.

Preference-then-Agent Sequential Priority Rule, $\boldsymbol{S P}^{p, q}$ : Let $p: N \rightarrow N$ be a one-to-one and onto function that provides agent priorities, and let $q: \mathcal{R} \rightarrow\{1,2, \cdots, n !\}$ be a one-to-one and onto function that provides preference priorities. For $R_{i} \in \mathcal{R}$, let $I\left(R_{i}\right) \equiv\left\{j \in N \mid R_{j}=R_{i}\right\}$. Given a profile $R$, the allocation $S P^{p, q}(R)$ is determined as follows.

Step 1: Let $i \in \operatorname{argmin}_{j \in N} q\left(R_{j}\right)$. If $\left|I\left(R_{i}\right)\right|=1$, agent $i$ obtains his most-preferred object in $K$. If $\left|I\left(R_{i}\right)\right| \geq 2$, agent $i^{\prime}=\operatorname{argmin}_{j \in I\left(R_{i}\right)} p(j)$ obtains his most-preferred object in $K$, agent $i^{\prime \prime}=\operatorname{argmin}_{j \in I\left(R_{i}\right) \backslash\left\{i^{\prime}\right\}} p(j)$ obtains his most-preferred object in $K \backslash\left\{S P_{i^{\prime}}^{p, q}(R)\right\}$, and so on.

Step 2: Let $l \in \operatorname{argmin}_{j \in N \backslash I\left(R_{i}\right)} q\left(R_{j}\right)$. We use the same procedure as in Step 1. We continue until no agents remain.

The next example illustrates the definition.

ExAmple 2: Let $n=3$ and let $K=\{a, b, c\}$. Let $p(1)=2, p(2)=1, p(3)=3, q(a R b R$ $c)=1$, and $q(a R c R b)=2, q(b R a R c)=3, q(b R c R a)=4, q\left(\begin{array}{lllll}c & R & a & R & R\end{array}\right)=5$, $q(c R b R a)=6$. Consider the following preference profiles:

$\begin{array}{llllll}R^{1} & q & R^{2} & q & R^{3} & q p \\ R_{1}^{1}: a \boldsymbol{c} b & 2 & R_{1}^{2}: \boldsymbol{a} b c & 1 & R_{1}^{3}: a \boldsymbol{b} c & 12 \\ R_{2}^{1}: \boldsymbol{a} b c & 1 & R_{2}^{2}: a \boldsymbol{c} b & 2 & R_{2}^{3}: \boldsymbol{a} b c & 11 \\ R_{3}^{1}: \boldsymbol{b} a c & 3 & R_{3}^{2}: \boldsymbol{b} a c & 3 & R_{3}^{3}: b a \boldsymbol{c} & 33 \\ 2 \rightarrow 1 \rightarrow 3 & & 1 \rightarrow 2 \rightarrow 3 & & 2 \rightarrow 1 \rightarrow 3\end{array}$

At $R^{1}$, all agents have distinct preferences, so only the preference priority $q$ is required to determine the allocation. The induced final priority sequence is agent 2 , agent 1 , and agent 3 , 
and the allocation is $S P^{p, q}\left(R^{1}\right)=(c, a, b)$. Similarly, $S P^{p, q}\left(R^{2}\right)=(a, c, b)$. At $R^{3}$, agents 1 and 2 have the same preferences, and thus, priority is determined by both $q$ and agent priority level $p$. According to $q$, agents 1 and 2 have higher priority than agent 3. According to $p$, agent 2 has higher priority than agent 1 . Therefore, the final priority sequence is agent 2 , agent 1 , and agent 3 , and the allocation is $S P^{p, q}\left(R^{3}\right)=(b, a, c)$.

In this example, agent 2 can benefit from misrepresenting his preference at $R^{2}$. There are many types of manipulations that work under this rule. We can infer from this observation that pairwise-anonymity on distinct profiles seems incompatible with strategy-proofness. As we show next, these two properties are incompatible.

Theorem 3: Let $n \geq 3$. Then, no rule is pairwise-anonymous on distinct profiles and strategyproof.

Proof: Let $f$ be a rule satisfying the axioms of the theorem. Let $R \in \mathcal{R}^{N}$ be such that $R_{1}=R_{2}$ and for each pair $\{i, j\} \subset N \backslash\{1\}, R_{i} \neq R_{j}$ and $t\left(R_{i}\right) \neq t\left(R_{j}\right)$. Without loss of generality, assume that $f_{2}(R) P_{1} f_{1}(R)$. Then, there is $R_{0} \in \mathcal{R}$ such that $L\left(R_{1}, f_{1}(R)\right) \varsubsetneqq L\left(R_{0}, f_{2}(R)\right)$ and $R_{0} \neq R_{i}$ for each $i \in N \backslash\{1\}$. Let $R^{\prime} \equiv\left(R_{0}, R_{2}, R_{-12}\right)$ and $R^{\prime \prime} \equiv\left(R_{1}, R_{0}, R_{-12}\right)$. By strategy-proofness, $f_{1}\left(R^{\prime}\right) \in L\left(R_{1}, f_{1}(R)\right)$. Thus, $L\left(R_{1}, f_{1}(R)\right) \varsubsetneqq L\left(R_{0}, f_{2}(R)\right)$ implies $f_{2}(R) P_{0} f_{1}\left(R^{\prime}\right)$. By pairwise-anonymity distinct profiles and $R_{1}=R_{2}, f_{2}\left(R^{\prime \prime}\right)=f_{1}\left(R^{\prime}\right)$. Therefore, $f_{2}\left(R_{2}, R_{-2}^{\prime \prime}\right) P_{2}^{\prime \prime} f_{2}\left(R^{\prime \prime}\right)$. This contradicts strategy-proofness.

Q.E.D.

\section{Independence of the axioms}

In this section, we demonstrate that no axiom in the theorems is redundant. Because we have already provided a rule satisfying pairwise-anonymity on distinct profiles and Pareto-efficiency, we next provide one rule satisfying pairwise-anonymity on distinct preferences, one satisfying independence of others' permutations, and one satisfying strategy-proofness.

(i) Pairwise-anonymity on distinct preferences.

Proposition 1 states that there is no rule that is pairwise-anonymous on distinct preferences when the number of agents is three, four, or five. In general, we have not been able to determine whether a rule is that is pairwiseanonymous on distinct preferences.

Proposition 1: Let $3 \leq n \leq 5$. Then, no rule is pairwise-anonymous on distinct preferences. Proof: (a) $n=3 \quad$ Pairwise-anonymity on distinct preferences implies independence of other's permutations. Thus, by Theorem 2, there is no rule that is pairwise-anonymous on distinct preferences.

(b) $n=4 \quad$ Let $f$ be pairwise-anonymous on distinct preferences. Let $R^{1} \in \mathcal{R}^{N}$ be such that $R_{1}^{1}=R_{2}^{1} \neq R_{3}^{1} \neq R_{4}^{1}$. We denote $f\left(R^{1}\right)=(a, b, c, d)$. Let $R^{2} \equiv\left(R_{3}^{1}, R_{2}^{1}, R_{-23}^{1}\right)$ be obtained from $R^{1}$ by transposing the preferences of agents 2 and 3 . Then, by pairwise-anonymity on distinct preferences, $f_{2,3}\left(R^{2}\right)=(c, b)$. Let $R^{3} \equiv\left(R_{4}^{1}, R_{2}^{1}, R_{-24}^{1}\right)$. Then, by pairwise-anonymity on distinct preferences, $f_{2,4}\left(R^{3}\right)=(d, b)$.

Here, $f_{1}\left(R^{2}\right) \in\{a, d\}$. Suppose that $f_{1}\left(R^{2}\right)=a$. Let $R^{4} \equiv\left(R_{2}^{2}, R_{1}^{2}, R_{-12}^{2}\right)$ and $R^{5} \equiv$ $\left(R_{3}^{1}, R_{1}^{1}, R_{-13}^{1}\right)$. Because $R_{1}^{1}=R_{2}^{1}, R^{4}=R^{5}$. However, by pairwise-anonymity on distinct preferences, $f_{2}\left(R^{4}\right)=a$ and $f_{3}\left(R^{5}\right)=a$. This contradicts feasibility. Thus, $f_{1}\left(R^{2}\right)=d$, and thus, $f\left(R^{2}\right)=(d, c, b, a)$.

By a similar argument, $f\left(R^{3}\right)=(c, d, a, b)$. Let $R^{6} \equiv\left(R_{4}^{2}, R_{3}^{2}, R_{-34}^{2}\right)$ and $R^{7} \equiv\left(R_{3}^{3}, R_{2}^{3}, R_{-23}^{3}\right)$. Because $R_{1}^{1}=R_{2}^{1}, R^{6}=R^{7}$. However, by pairwise-anonymity on distinct preferences, $f_{3}\left(R^{6}\right)=$ 
$a$ and $f_{2}\left(R^{7}\right)=a$. This contradicts feasibility.

(c) $n=5 \quad$ Let $f$ be pairwise-anonymous on distinct preferences. Let $R \in \mathcal{R}^{N}$ be such that $R_{1}=R_{2}=R_{3}=R_{4} \neq R_{5}$. We denote $f(R) \equiv(a, b, c, d, e)$. Let $R^{1} \equiv\left(R_{5}, R_{2}, R_{3}, R_{4}, R_{1}\right)$, $R^{2} \equiv\left(R_{1}, R_{5}, R_{3}, R_{4}, R_{2}\right), R^{3} \equiv\left(R_{1}, R_{2}, R_{5}, R_{4}, R_{3}\right)$, and $R^{4} \equiv\left(R_{1}, R_{2}, R_{3}, R_{5}, R_{4}\right)$ be obtained by transposing two preferences of $R$. Then, by pairwise-anonymity on distinct preferences, $f_{1,5}\left(R^{1}\right)=(e, a), f_{2,5}\left(R^{2}\right)=(e, b), f_{3,5}\left(R^{3}\right)=(e, c)$, and $f_{4,5}\left(R^{4}\right)=(e, d)$.

Suppose that $f_{2}\left(R^{1}\right)=b$. Then, by pairwise-anonymity on distinct preferences, $f_{1}\left(R^{2}\right)=b$ because $R^{2}$ can be obtained from $R^{1}$ by transposing $R_{1}^{1}$ and $R_{2}^{1}$. However, $f_{5}\left(R^{2}\right)=b$, which is a contradiction. Therefore, $f_{2}\left(R^{1}\right) \neq b$. Similarly $f_{3}\left(R^{1}\right) \neq c$ and $f_{4}\left(R^{1}\right) \neq d$. Thus, $f\left(R^{1}\right)=(e, c, d, b, a)$ or $(e, d, b, c, a)$.

Let $f\left(R^{1}\right)=(e, c, d, b, a)$. Then, by pairwise-anonymity on distinct preferences, $f_{1}\left(R^{2}\right)=c$ because $R^{2}$ can be obtained from $R^{1}$ by transposing $R_{1}^{1}$ and $R_{2}^{1}$. Similarly, $f_{1}\left(R^{3}\right)=d$. At $R^{2}$, either agents 3 or 4 must be assigned $d$. Assume that $f_{3}\left(R^{2}\right)=d$. Then, pairwise-anonymity on distinct preferences implies $f_{2}\left(R^{3}\right)=d$. However, we have already obtained that $f_{1}\left(R^{3}\right)=d$. This is a contradiction. We have the same contradiction even if agent 4 is assigned $d$ at $R$, and this means that if $f\left(R^{1}\right)=(e, c, d, b, a)$, a contradiction occurs. Similarly, we can derive a contradiction even if $f\left(R^{1}\right)=(e, d, b, c, a)$.

Q.E.D.

(ii) Independence of others' permutations. We show an example of a rule that is independent of others' permutations but not pairwise-anonymous on distinct preferences. Let $q: \mathcal{R} \rightarrow\{1,2, \cdots, n !\}$ be a one-to-one and onto function that gives preference priorities. The rule is as follows: If all agents have distinct preferences, each agent obtains his most-preferred object according to his preference priority $q$. This process is the same as that of the Preferencethen-Agent sequential priority rule, except that we do not use agent priority $p$. If several agents have the same preferences, we use the constant rule, meaning that all allocations are the same for each such profile. Formally,

$$
f(R)= \begin{cases}\operatorname{constant}(R) & \text { if } \exists i, j \in N \text { such that } R_{i}=R_{j} \\ S P^{q}(R) & \text { otherwise }\end{cases}
$$

(iii) Strategy-proofness. The rule defined next is strategy-proof but not pairwise-anonymous on distinct profiles. ${ }^{13}$

Sequential priority rule: Let $\pi: N \rightarrow N$. For each $R \in \mathcal{R}^{N}$, agent $\pi(1)$ obtains his most preferred object in $K$, agent $\pi(2)$ obtains his most preferred object in $K \backslash\left\{f_{\pi(1)}(R)\right\}$, agent $\pi(3)$ obtains his most preferred object in $K \backslash\left\{f_{\pi(1)}(R), f_{\pi(2)}(R)\right\}$, and so on.

\section{Summary and concluding remarks}

In this section, we provide a summary table of our properties and some remarks on our results.

In the following table, Eff, STP, and IOP respectively indicate Pareto-efficiency, strategyproofness, and independence of others' permutations. "+" means that the two properties are compatible, and "-" means that they are incompatible. "?" means that it is unknown to us whether the two properties are compatible.

\footnotetext{
${ }^{13}$ For example, consider the case in which agent 1 has highest priority, agent 2 has the lowest priority, and $a P_{1} b P_{1} \cdots$ and $a P_{2} c P_{2} \cdots$, where $a, b, c \in K$.
} 


\begin{tabular}{c|cccc}
\hline & existence & Eff & STP & IOP \\
\hline Anonymity on distinct preferences & - & - & - & - \\
Pairwise-anonymity on distinct preferences & $?$ & - & - & - \\
Pairwise-anonymity on distinct profiles & + & + & - & + \\
\hline
\end{tabular}

(i) Introducing the null object. In our model, the number $n$ of agents and the number $m$ of objects are equal. If $n<m$, all of our results still hold except for Fact $2 .^{14}$

If $n>m$, some agents inevitably receive a "null object," which means that they obtain nothing. In this case, there are rules satisfying standard anonymity and strategy-proofness. A trivial example of such rules is constant rules in which all agents receive the null object. In the case where preferences ranking the null object higher than some real objects are admissible, Kesten and Yazici (2012) analyze nontrivial rules satisfying standard anonymity and strategyproofness.

The case that has not yet been analyzed is that where $n>m$ and all real objects are desirable, that is, all real objects are preferred to the null object. We discuss the difficulty of this case.

First, if $n$ is substantially greater than $m$, our notions of anonymity are weaker or even completely meaningless. For example, assume that $n=7$ and $m=3$. Then, an agent's preference is chosen among six preferences so that in each preference profile, at least two agents have the same preferences. It follows that pairwise-anonymity of distinct profiles requires nothing. Although the other notions of anonymity require something, the number of admissible permutations decreases so that these notions will be weaken.

There are other difficulties in characterizing rules satisfying anonymity requirements. For example, if $n=7$ and $m=3$, there are four agents who must obtain the null object. Recall that the key point of our proofs of characterizations are that two agents with the same preferences must receive distinct objects. This point fails to hold, so our proofs do not work.

Owing to these difficulties, we leave the case where $n>m$ and all real objects are desirable for future research.

(ii) Characterization of pairwise-anonymous on distinct profiles and Pareto-efficient rules. As we demonstrated in Section 4, there are rules satisfying pairwise-anonymity on distinct profiles and Pareto-efficiency. They are the Preference-then-Agent sequential priority rules. Other rules satisfying the two properties exist. Consider the following example:

$$
f(R)= \begin{cases}S P^{p, q}(R) & \text { if } \exists i, j \in N \text { such that } R_{i}=R_{j} \\ S P^{q}(R) & \text { otherwise }\end{cases}
$$

where $S P^{q}$ is a simple sequential priority rule with agent priority $q$. This rule also satisfies both pairwise-anonymity on distinct profiles and Pareto-efficiency. The answer to which class of rules satisfies these two conditions remains unknown.

(iii) The strongest condition of anonymity.

In this paper, we proposed several variants of the anonymity condition. For each variant, a class of preference profiles is specified, and we

\footnotetext{
${ }^{14}$ If $n<m$, choose $n$ objects among $m$, and then apply the rule for the example in Section 5 (ii) to the $n$ objects. This rule satisfies pairwise-anonymous on distinct profiles and independent of others' permutations, but does not respect consumer sovereignty.
} 
permute preferences of profiles only if the profile belongs to the class. Note that the smaller the specified class of preference profiles is, the weaker the corresponding anonymity condition is. For example, consider preference profiles such that for each pair $\{i, j\} \subset N, t\left(R_{i}\right) \neq t\left(R_{j}\right)$, that is, it is possible for each agent to receive his favorite object. We call such profiles "no-conflict profiles." The requirement of anonymity on only no-conflict profiles is sufficiently weak that it is compatible with both Pareto-efficiency and strategy-proofness. Determining the conditions where the strongest variant of anonymity is compatible with these two properties is of interest.

When $n=3$, anonymity requirement on the class of no-conflict profiles is the strongest variant compatible with the two properties. That is, if we impose anonymity requirement on some profiles in addition to no-conflict profiles, no rule satisfies this anonymity condition along with Pareto-efficiency and strategy-proofness. We sketch the proof of this claim.

Let $R^{1}, R^{2}$ and $R^{3} \in \mathcal{R}^{N}$ be as follows:

$$
\begin{array}{lll}
R^{1} & R^{2} & R^{3} \\
R_{1}^{1}: a b c & R_{1}^{2}: a c b & R_{1}^{3}: a b c \\
R_{2}^{1}: a b c & R_{2}^{2}: a b c & R_{2}^{3}: a c b \\
R_{3}^{1}: b a c & R_{3}^{2}: b a c & R_{3}^{3}: b a c
\end{array}
$$

where $R^{2}$ and $R^{3}$ are the only profiles to which the anonymity condition can be applicable other than no-conflict profiles. By Pareto-efficiency, either agents 1 or 2 receives $a$ at $R^{1}$. Without loss of generality, let $f_{1}\left(R^{1}\right)=b$ or $c$ and $f_{2}\left(R^{1}\right)=a$. Then, by strategy-proofness, $f_{1}\left(R^{2}\right)=b$ or $c$. Furthermore, by the anonymity condition, $f_{2}\left(R^{3}\right)=b$ or $c$. However, this contradicts strategy-proofness because $f_{2}\left(R^{1}\right) R_{2}^{3} f_{2}\left(R^{3}\right)$. We can derive the same contradiction using similar argument in the other cases.

Determining which conditions are the strongest variant of anonymity together with Paretoefficiency and strategy-proofness when $n>3$ remains unanswered.

\section{References}

Abdulkadiroglu, A and Sonmez, T (1999) House allocation with existing tenants, Journal of Economic Theory, Volume 88, Issue 2, 233-260.

Bogomolnaia, A and Moulin, H (2001) A new solution to the random assignment problem, Journal of Economic Theory, Volume 100, Issue 2, 295-328.

Ehlers, L (2002) Coalitional strategy-proof house allocation, Journal of Economic Theory, Volume 105, Issue 2, 298-317.

Hatfield, J W (2009) Strategy-proof, efficient, and nonbossy quota allocations, Social Choice and Welfare, Volume 33, Issue 3, 505-515.

Kesten, O and Kurino, M (2013) Do outside options matter in school choice? A new perspective on the efficiency vs. strategy-proofness trade-off, mimeo.

Kesten, O and Yazici, A (2012) The Pareto dominant strategy-proof and equitable rule for problems with indivisible goods, Economic Theory, Volume 50, Number 2, 463-488. 
Ma, J (1994) Strategy-proofness and the strict core in a market with indivisibilities, International Journal of Game Theory, Volume 23, Issue 1, 75-83.

Miyagawa, E (2002) Strategy-proofness and the core in house allocation problems, Games and Economic Behavior, Volume 38, Issue 2, 347-361.

Papai, S (2000a) Strategyproof assignment by hierarchical exchange, Econometrica, Volume 68, Issue 6, 1403-1433.

Papai, S (2000b) Strategyproof multiple assignment using quotas, Review of Economic Design, Volume 5, Issue 1, 91-105.

Shapley, L and Scarf, H (1974) On cores and indivisibility, Journal of Mathematical Economics, Volume 1, Issue 1, 23-37.

Svensson, L (1999) Strategy-proof allocation of indivisible goods, Social Choice and Welfare, Volume 16, Issue 4, 557-567.

Takaymiya, K (2001) Coalition Strategy-proofness and monotonicity in Shapley-Scarf housing markets, Mathematical Social Sciences, Volume 41, Issue 2, 201-213.

Thomson, W (2012) On the axiomatics of resource allocation: interpreting the consistency principle, Economics and Philosophy, Volume 28, Issue 3, 385-421.

Zhou, L (1990) On a conjecture by Gale about one-sided matching problems, Journal of Economic Theory, Volume 52, Issue 1, 123-135. 